\title{
Effect of Deuterium-Depleted Water on Selected Cardiometabolic Parameters in Fructose-Treated Rats
}

\author{
R. REHAKOVA ${ }^{1}$, J. KLIMENTOVA ${ }^{1}$, M. CEBOVA ${ }^{1}$, A. BARTA ${ }^{1}$, Z. MATUSKOVA ${ }^{1}$, \\ P. LABAS ${ }^{2}$, O. PECHANOVA ${ }^{1}$ \\ ${ }^{1}$ Institute of Normal and Pathological Physiology, Slovak Academy of Sciences, Bratislava, Slovak \\ Republic, ${ }^{2}$ First Department of Surgery, Faculty of Medicine, Comenius University, Bratislava, \\ Slovak Republic
}

Received July 20, 2016

Accepted September 5, 2016

\section{Summary}

Deuterium-depleted water (DDW) has a lower concentration of deuterium than occurs naturally (less than $145 \mathrm{ppm}$ ). While effects of DDW on cancer started to be intensively studied, the effects on cardiovascular system are completely unknown. Thus, we aimed to analyze the effects of DDW ( $55 \pm 5 \mathrm{ppm})$ administration to 12-week-old normotensive Wistar-Kyoto rats (WKY) and spontaneously hypertensive rats (SHR) treated with $15 \%$ fructose for 6 weeks. Blood pressure (BP) and selected biochemical parameters were measured together with determination of nitric oxide synthase (NOS) activity and iNOS and eNOS protein expressions in the left ventricle (LV) and aorta. Neither DDW nor fructose had any significant effect on BP in both strains. DDW treatment decreased total cholesterol and triglyceride levels in WKY, but it was not able to prevent increase in the same parameters elevated due to fructose treatment in SHR. Both fructose and DDW increased insulin level in WKY. Fructose did not affect NOS activity either in WKY or SHR. DDW increased NOS activity in LV of both WKY and SHR, while it decreased NOS activity and iNOS expression in the aorta of SHR with or without fructose treatment. In conclusion, DDW treatment significantly modified biochemical parameters in WKY together with NOS activity elevation in the heart. On the other hand, it did not affect biochemical parameters in SHR, but decreased NOS activity elevated due to iNOS upregulation in the aorta.

\section{Key words}

Deuterium-depleted water • Fructose • Nitric oxide • Plasma lipids • Insulin

\section{Corresponding author}

O. Pechanova, Institute of Normal and Pathological Physiology, Slovak Academy of Sciences, Sienkiewiczova 1, 81371 Bratislava, Slovak Republic. E-mail: olga.pechanova@savba.sk

\section{Introduction}

Deuterium-depleted water (DDW), also known as light water, is water which has a lower concentration of deuterium than occurs naturally (less than $145 \mathrm{ppm}$ ). In nature the ratio of deuterium to hydrogen $(\mathrm{D} / \mathrm{H})$ is about 1:6600; this means that the natural concentration of $\mathrm{D}$ is about $150 \mathrm{ppm}(0.015$ atom \%). The natural water consists from a mixture of $\mathrm{H}_{2} \mathrm{O}$ and $\mathrm{D}_{2} \mathrm{O}$ and the natural levels of D in terrestrial waters vary from 14 to $150 \mathrm{ppm}$ (Friedman et al. 1964, Stefanescu et al. 2003). In the bottled water, concentrations of deuterium typically vary between 135 and $158 \mathrm{ppm}$; this means over $16 \mathrm{mmol} / \mathrm{l}$ (Katz et al. 1962). DDW can be obtained by continuous vacuum distillation of natural water, but often it represents just a by-product of the separation process for obtaining heavy water (Mladin et al. 2014). In living organisms D concentration exceeds $10 \mathrm{mmol} / \mathrm{l}$, which is above the range of some vital mineral levels (Laissue et al. 1983). Deuterium in human plasma is redundant with concentrations reaching $12-14 \mathrm{mmol} / \mathrm{l}$ in comparison with calcium (2.24-2.74 $\mathrm{mmol} / \mathrm{l})$, potassium (5.0-5.1 $\mathrm{mmol} / \mathrm{l})$, and glucose (3.3-6.1 $\mathrm{mmol} / \mathrm{l})$ circulating concentrations (Laissue et al. 1983). Concentration of deuterium in the body water correlates well with the deuterium level in the environment (Gross and Spindel 
1962). When DDW is administrated for a long period, it may reduce the concentration of deuterium throughout the body, thus activating cellular mechanisms which are depending on protons (Mladin et al. 2014).

The scientific literature on the effects of deuterium depletion on living cells has been very limited - some 70 peer-reviewed research papers were available via PubMed in mid-2016. However, the research developed by specialized institutions revealed different bioactive effects of DDW on living organisms with main effects on tumor cell line remission (for review see Boros et al. 2016). Other effects include immunity stimulation, increase of animal resistance to sublethal and lethal doses of radiation, dermatological diseases, and positive effects upon the leukocytes and erythrocytes (Buzgariu et al. 1997, Haulica et al. 1998, Haulica et al. 2003). Yet, longlasting effects of DDW on cardiovascular parameters have never been investigated. Since the study of Lisitsyn et al. (2014) indicated positive effects of DDW on some metabolic parameters, we aimed to investigate longlasting effects of DDW on lipid profile, glucose and insulin plasma concentrations, blood pressure, and nitric oxide (NO) generation in the heart and aorta in normotensive Wistar-Kyoto rats (WKY) and spontaneously hypertensive rats (SHR) treated with $15 \%$ fructose for six weeks.

\section{Methods}

\section{Chemicals and drugs}

DDW was a general gift from Deuterium-D2 Ltd., Slovakia. All chemicals used were purchased from Sigma Chemicals Co. (Germany) when not specified.

\section{Animals and treatment}

The experiment was carried out on 48 male 12-week-old rats ( $n=24 \mathrm{WKY}$ and $\mathrm{n}=24$ SHR). Six WKY and SHR were taken as controls and drank tap water, whereas the remaining rats $(n=6$ in each group) were given free access to DDW ( $55 \pm 5 \mathrm{ppm})$ and $15 \%$ solution of fructose in the drinking or deuterium-depleted water for 6 weeks. All animals were kept under standard laboratory conditions (12-h light/darkness cycle) in a room with controlled temperature $\left(21 \pm 1{ }^{\circ} \mathrm{C}\right)$. Animals were fed a standard commercial chow diet ad libitum. All procedures and experimental protocols were approved by the Ethical Committee of the Institute of Normal and Pathological Physiology SAS.

Systolic blood pressure (SBP) was measured by tail-cuff plethysmography every week. At the end of experiment the animals were sacrificed under $\mathrm{CO}_{2}$ anesthesia and body weight (BW) and heart weight (HW) were determined. Samples of the blood were collected immediately for determination of plasma lipid profile, glucose and insulin concentrations. Samples of the heart left ventricle (LV) and aorta were used for determination of NOS activity and Western blot analysis.

\section{Biochemical parameters}

Lipid profile (total cholesterol, triglycerides, high-density lipoprotein cholesterol) and plasma glucose and insulin concentrations were determined by commercially available kits (Crystal Chem, USA).

\section{Total NOS activity}

Total NOS activity was determined in crude homogenates of $\mathrm{LV}$ and aorta by measuring $\mathrm{L}-\left[{ }^{3} \mathrm{H}\right]$ citrulline formation from $\mathrm{L}-\left[{ }^{3} \mathrm{H}\right]$ arginine (MP Biochemicals, USA) as described elsewhere (Pechanova et al. 2007). NOS activity was expressed as $\mathrm{pkat} / \mathrm{min}$ per gram of protein.

\section{Western blot analysis}

Samples of the LV and aorta were probed with polyclonal rabbit antibodies: endothelial NOS (eNOS) (Abcam, England) and inducible NOS (iNOS) (Abcam, England). Bound antibody was detected using a secondary peroxidase-conjugated anti-rabbit antibody (Abcam, Encland). The bands were visualized using the enhanced chemiluminescence system (ECL, Amersham, UK) and analyzed densitometrically using Photo-Capt V.99 software.

\section{Statistical analysis}

All results are expressed as means \pm SEM. Analysis of variance and Duncan test were used for the statistical analysis. Due to lot of data and for better orientation in the tables and figures, groups of the same strain were compared only. Values were considered to differ significantly if the probability value was less than $\mathrm{p}<0.05$.

\section{Results}

\section{Systolic blood pressure and weight ratios}

Neither DDW nor fructose had any significant effect on SBP and relative heart weight in WKY or SHR (Table 1). 
Table 1. Effects of deuterium-depleted water and fructose in drinking or deuterium depleted water on systolic blood pressure, body weight and relative heart weight.

\begin{tabular}{lcccc}
\hline & SBP (mm Hg) & BW (g) & HW (mg) & HW/BW (mg/g) \\
\hline$W K Y$ & $119.6 \pm 1.33$ & $443 \pm 17$ & $1102 \pm 23$ & $2.5 \pm 0.04$ \\
$W K Y-D D W$ & $122.3 \pm 5.06$ & $455 \pm 16$ & $1136 \pm 25$ & $2.5 \pm 0.06$ \\
$W K Y+F R U$ & $124.3 \pm 2.79$ & $451 \pm 11$ & $1095 \pm 27$ & $2.4 \pm 0.03$ \\
$W K Y-D D W+F R U$ & $121.8 \pm 1.74$ & $446 \pm 9$ & $1115 \pm 36$ & $2.5 \pm 0.05$ \\
& & & & \\
SHR & $193.5 \pm 5.96$ & $362 \pm 5$ & $1270 \pm 29$ & $3.5 \pm 0.08$ \\
SHR-DDW & $194.8 \pm 2.27$ & $356 \pm 6$ & $1250 \pm 34$ & $3.5 \pm 0.10$ \\
SHR $+F R U$ & $196.7 \pm 2.78$ & $369 \pm 8$ & $1305 \pm 28$ & $3.5 \pm 0.06$ \\
SHR-DDW $+F R U$ & $200.8 \pm 0.75$ & $374 \pm 8$ & $1287 \pm 32$ & $3.4 \pm 0.05$ \\
\hline
\end{tabular}

Data are means \pm SEM from 6 animals in each group. WKY - Wistar-Kyoto rats, SHR - spontaneously hypertensive rats, SBP - systolic blood pressure, BW - body weight, HW - heart weight, DDW - deuterium-depleted water, FRU - fructose.

Table 2. Effects of deuterium-depleted water and fructose in drinking or deuterium depleted water on total cholesterol, triglycerides, high-density lipoprotein cholesterol, glucose and insulin levels.

\begin{tabular}{llllll}
\hline & CHOL (mmol/l) & TGs (mmol/l) & HDL (mmol/l) & GLU (mmol/l) & Insulin (mmol/l) \\
\hline$W K Y$ & $1.93 \pm 0.08$ & $1.25 \pm 0.10$ & $1.62 \pm 0.03$ & $13.27 \pm 1.15$ & $3.59 \pm 0.16$ \\
$W K Y-D D W$ & $1.65 \pm 0.07^{¥}$ & $0.89 \pm 0.08^{¥}$ & $1.56 \pm 0.05$ & $13.27 \pm 1.14$ & $6.28 \pm 0.93^{¥}$ \\
$W K Y+F R U$ & $1.89 \pm 0.10$ & $1.48 \pm 0.26$ & $1.61 \pm 0.09$ & $15.30 \pm 1.68$ & $8.09 \pm 0.86^{¥}$ \\
$W K Y-D D W+F R U$ & $1.58 \pm 0.06^{¥+}$ & $1.59 \pm 0.21$ & $1.45 \pm 0.06$ & $13.95 \pm 1.83$ & $4.64 \pm 0.32^{¥+}$ \\
& & & & & \\
SHR & $1.24 \pm 0.06$ & $0.71 \pm 0.03$ & $1.12 \pm 0.06$ & $11.28 \pm 1.06$ & $6.78 \pm 0.58$ \\
SHR-DDW & $1.25 \pm 0.06$ & $0.72 \pm 0.07$ & $1.09 \pm 0.04$ & $14.32 \pm 0.53$ & $6.52 \pm 0.94$ \\
SHR $+F R U$ & $1.49 \pm 0.04^{*}$ & $1.60 \pm 0.16^{*}$ & $1.09 \pm 0.04$ & $14.84 \pm 0.84$ & $4.82 \pm 0.57^{*}$ \\
SHR-DDW+FRU & $1.48 \pm 0.06^{*}$ & $1.46 \pm 0.16^{*}$ & $1.24 \pm 0.04^{*}$ & $13.84 \pm 1.14$ & $4.63 \pm 0.39^{*}$ \\
\hline
\end{tabular}

Data are means \pm SEM from 6 animals in each group. WKY - Wistar-Kyoto rats, SHR - spontaneously hypertensive rats, FRU fructose, $\mathrm{CHOL}$ - total cholesterol, TGs - triglycerides, $\mathrm{HDL}$ - high-density lipoprotein cholesterol, GLU - glucose. $¥ \mathrm{p}<0.05$ compared to $W K Y,+p<0.05$ compared to WKY+FRU, $* p<0.05$ compared to SHR.

\section{Biochemical parameters}

Fructose treatment increased total cholesterol and triglyceride levels in SHR only. DDW treatment decreased total cholesterol and triglyceride levels in WKY, while no changes were observed in SHR. DDW treatment was not able to prevent the increase in total cholesterol and triglyceride levels elevated due to fructose treatment in SHR. However, in the same strain it increased HDL cholesterol level in fructose-treated rats (Table 2).

Neither fructose nor DDW treatment affected glucose levels in WKY and SHR. Both fructose and DDW increased insulin level in WKY, while fructose decreased it in SHR. Interestingly, in WKY simultaneous fructose and DDW treatment decreased insulin level compared to fructose treatment alone (Table 2).

\section{Total NOS activity}

Fructose treatment had no effect on NOS activity either in WKY or SHR. DDW increased significantly NOS activity in LV of both WKY and SHR. Concomitant treatment of DDW with fructose increased NOS activity in LV of SHR and increasing tendency was also seen in WKY. On the other hand, DDW treatment decreased NOS activity in the aorta of SHR with or without fructose treatment (Figs 1A and 1B). 

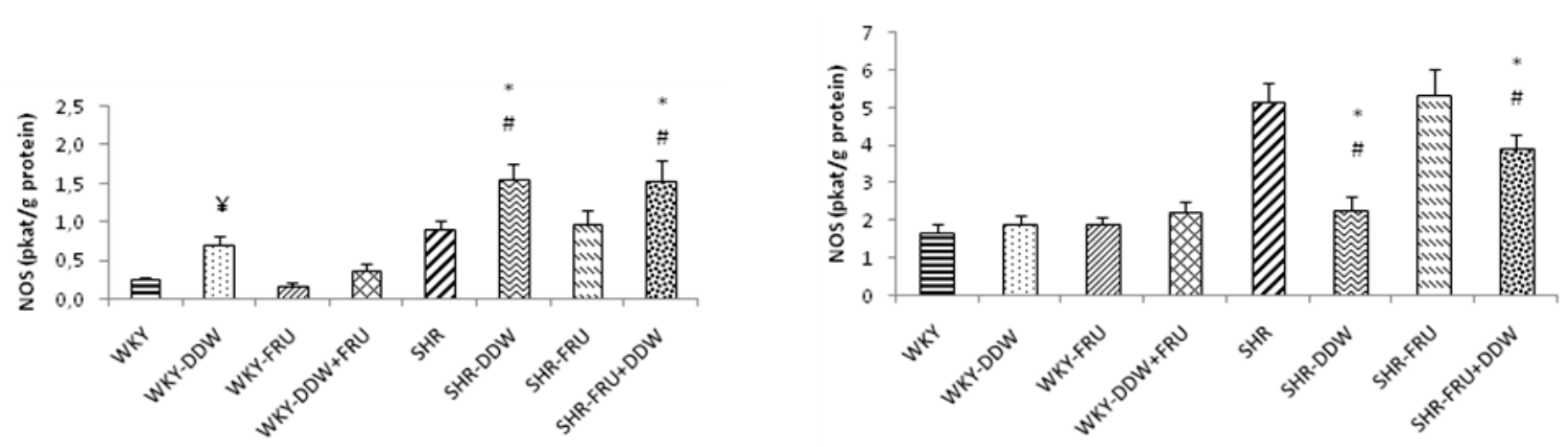

Fig. 1. Effect of deuterium-depleted water (DDW) and fructose (FRU) treatment on nitric oxide synthase (NOS) activity in the heart (left panel) and the aorta (right panel) of Wistar-Kyoto (WKY) and spontaneously hypertensive rats (SHR). Data are means \pm SEM from 6 animals in each group. $¥ p<0.05$ compared to WKY, * $p<0.05$ compared to SHR, \# $p<0.05$ compared to SHR-FRU.

(A)

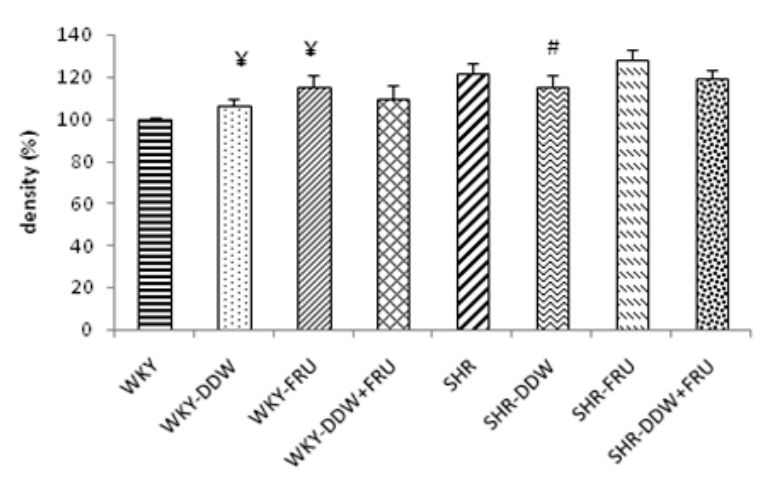

(C)

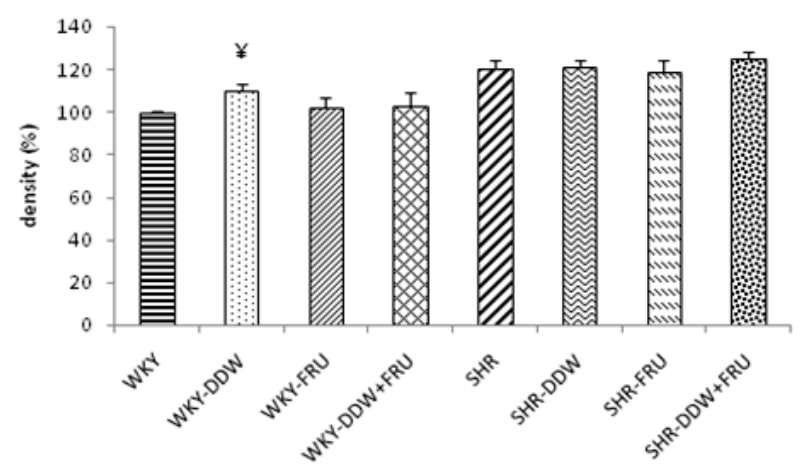

(B)

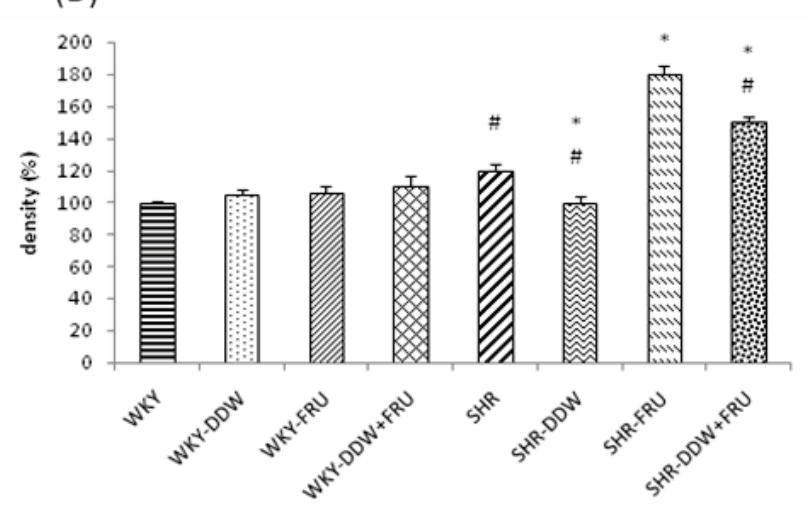

(D)

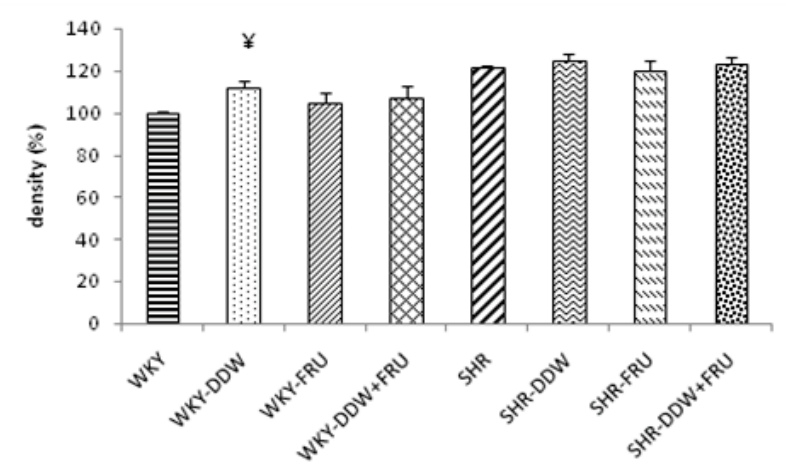

Fig. 2. Effect of deuterium-depleted water (DDW) and fructose (FRU) treatment on protein iNOS expression in the left ventricle (A) and the aorta (B) and eNOS expression in the left ventricle (C) and the aorta (D) of Wistar-Kyoto (WKY) and spontaneously hypertensive rats (SHR). Data are means \pm SEM from 6 animals in each group. $¥ p<0.05$ compared to WKY, $* p<0.05$ compared to SHR, \# p<0.05 compared to SHR-FRU.

In the LV, iNOS expression was significantly increased after DDW treatment in WKY, while it was decreased in SHR (Fig. 2A). Similarly to NOS activity, DDW treatment decreased iNOS protein expression in the aorta of control and fructose-treated SHR (Fig. 2B). DDW treatment increased eNOS expression in the LV and aorta of WKY (Figs 2C and 2D).

\section{Discussion}

According to our best knowledge, the effect of DDW on NO generation in the cardiovascular system of rats has been analyzed for the first time. Similarly, in vivo DDW effects on selected biometrical and biochemical parameters have been shown for the first time.

Regarding blood pressure and relative heart 
weight, our results did not show any significant differences between the groups which received fructose in tap water or deuterium-depleted water. As expected, both parameters were significantly higher in SHR compared to WKY. These findings are in agreement with our (Pechanova et al. 2007, Simko et al. 2009) and other reports (Čejka et al. 2004, Firbas et al. 2005).

To see the effects of DDW on selected metabolic parameters like total plasma cholesterol, triglycerides, glucose and insulin, we treated the normotensive and spontaneously hypertensive rats with $15 \%$ fructose solution which was expected to alter these parameters. Fructose treatment increased total cholesterol and triglyceride levels in SHR only and did not affect glucose levels in either strain. Similarly to our experiment, chronic fructose intake, which increased plasma triglyceride level did not affect blood pressure of hereditary hypertriglyceridemic rats (Zicha et al. 2006, Kadlecová et al. 2004). In our study insulin level was increased after fructose treatment in WKY, while it was decreased in SHR. Commonly, a high flux of fructose to the liver, the main organ capable of metabolizing this carbohydrate, leads to a significantly enhanced rate of de novo lipogenesis and triglyceride synthesis, driven by the high flux of glycerol and acyl portions of triglyceride molecules from fructose catabolism. These metabolic disturbances appear to underlie the induction of insulin resistance often observed with high fructose intake in both humans and animal models (for review see Basciano et al. 2005). In our experiment, the administration of $15 \%$ fructose solution was probably not sufficient enough to increase total cholesterol and triglyceride levels in WKY. However, it increased these parameters in SHR with a higher insulin level compared to WKY. Previous results have shown that SHR are insulin-resistant and hyperinsulinemic. A decreased expression of insulin receptor in the liver of SHR provides a possible explanation for the insulin resistance and decreased insulin clearance present in this strain (Reaven and Chang 1991). Interestingly, in our experiment, fructose treatment decreased insulin level in SHR strain. Our results are in agreement with those of Hao et al. (2007) who found a direct link between elevated serum cholesterol and reduced insulin secretion, with normal secretion restored by cholesterol depletion. These authors further demonstrated that excess cholesterol inhibits insulin secretion by downregulation of neuronal NOS dimerization leading to a decrease in NO production. In the study of El-Bassossy et al. (2016), 12-week $10 \%$ fructose treatment of rats led to increased cholesterol level and hyperinsulinemia associated with decreased NO production in the aorta and impaired endotheliumdependent relaxation. On the other hand, recent in vivo mice studies demonstrated anti-atherogenic effects of insulin (Mori et al. 2016). Actually, insulin is a known activator of endothelial NOS, leading to increased production of $\mathrm{NO}$, which has potent anti-atherogenic effects (for review see $\mathrm{Yu}$ et al. 2011). In male apolipoprotein E-null mice with high cholesterol diet $(1.25 \%)$ insulin treatment $(0.05 \mathrm{U} /$ day $)$ increased both plasma NO levels and eNOS phosphorylation while decreased iNOS expression (Mori et al. 2016). In our experiment, however, fructose treatment did not affect NOS activity in either WKY or SHR regardless insulin level in these strains. Nevertheless, it upregulated significantly iNOS expression in the left ventricle of WKY and in the aorta of SHR. These changes probably would be mirrored in increased NOS activity after prolongation of fructose treatment. Similarly, Babacanoglu et al. (2013) found increased iNOS expression in the aorta of rats after $20 \%$ fructose solution treatment.

In our experimental study DDW decreased total cholesterol and triglyceride levels along with increased plasma insulin in WKY, which was associated with elevated NOS activity in the left ventricle. According to our experiments, both increased eNOS and iNOS expressions may contribute to this elevation of NOS activity. On the other hand, DDW treatment decreased insulin level elevated due to fructose treatment in this particular strain. In SHR, DDW treatment did not affect measured biochemical parameters, but it decreased NOS activity and iNOS expression in the aorta of SHR with or without fructose treatment. Mechanisms proposed in DDW particular effects emphasize the terminal complex of mitochondrial electron transport chain reducing molecular oxygen to deuterium-depleted water. This may potentially affect gluconeogenesis as well as fatty acid oxidation (for review see Boros et al. 2016, Somlyai et al. 2010). Preventive effects of DDW on lipid oxidation may be potentially associated with the good effects on reactive oxygen species and NO balance (Pechanova and Simko 2007, 2009, Vrankova et al. 2016).

Taken together, although DDW treatment showed different effects depending on rat strain and fructose treatment, generally in normotensive rats it was able to decrease total cholesterol and triglyceride levels along with increased insulin and NOS activity. On the other hand, in hypertensive rats, it may decrease NOS activity elevated due to iNOS upregulation showing 
an indication of anti-inflammatory potential.

\section{Conflict of Interest}

There is no conflict of interest.

\section{Acknowledgements}

This study was elaborated within the projects APVV0742-10, APVV-14-0932, VEGA 2/0195/15 and 2/0144/14.

\section{References}

BABACANOGLU C, YILDIRIM N, SADI G, PEKTAS MB, AKAR F: Resveratrol prevents high-fructose corn syrupinduced vascular insulin resistance and dysfunction in rats. Food Chem Toxicol 60: 160-167, 2013.

BASCIANO H, FEDERICO L, ADELI K: Fructose, insulin resistance, and metabolic dyslipidemia. Nutr Metab (Lond) 2: 5, 2005. doi: 10,1186/1743-7075-2-5.

BOROS LG, D'AGOSTINO DP, KATZ HE, ROTH JP, MEUILLET EJ, SOMLYAI G: Submolecular regulation of cell transformation by deuterium depleting water exchange reactions in the tricarboxylic acid substrate cycle. Med Hypothes 87: 69-74, 2016.

BUZGARIU W, CALOIANU M, LAZAR S: Structural and ultrastructural changes in fish spleen induced by heavy water. Rom J Biol Sci 1: 5-6, 1997.

ČEJKA J, ŽELEZNÁ B, VELEK J, ZICHA J, KUNEŠ J: LVV-hemorphin-7 lowers blood pressure in spontaneously hypertensive rats: Radiotelemetry study. Physiol Res 53: 603-607, 2004.

EL-BASSOSSY H, BADAWY D, NEAMATALLAH T, FAHMY A: Ferulic acid, a natural polyphenol, alleviates insulin resistance and hypertension in fructose fed rats: Effect on endothelial-dependent relaxation. Chem Biol Interact 254: 191-197, 2016.

FIRBAS U, MICHLITS W, AHARINEJAD S: Venous sphincters in the choroidea of spontaneously hypertensive rats. Anat Rec 284A: 537-543, 2005.

FRIEDMAN I, SCHOEN A, HARRIS B, JOSEPH J: The variation of the deuterium content of natural waters in the hydrologic cycle. Rev Geophys 2: 177-224, 1964.

GROSS PR, SPINDEL W: Heavy water inhibition of cell division: an approach to mechanism. Ann NY Acad Sci 90: 500-522, 1962.

HAO M, HEAD WS, GUNAWARDANA SC, HASTY AH, PISTON DW: Direct effect of cholesterol on insulin secretion: a novel mechanism for pancreatic beta-cell dysfunction. Diabetes 56: 2328-2338, 2007.

HAULICA I, PECULEA A, STEFANESCU I, TITESCU G, TODIRAS M, BILD W: Effects of heavy and deuteriumdepleted water of vascular reactivity. Rom J Physiol 35: 25-32, 1998.

HAULICA I, STEFANESCU I, BILD W, TITESCU G, TAMAIAN R, NASTASA V, IONITA T: Research concerning physiological effects of deuterium-depleted water on unspecific imunity. 2003. http://www.cdep.ro/pls/ proiecte/upl_pck.proiect?idp=7433.

KADLECOVÁ M, ČEJKA J, ZICHA J, KUNEŠ J: Does Cd36 gene play a key role in disturbed glucose and fatty acid metabolism in Prague hypertensive hypertriglyceridemic rats? Physiol Res 53: 265-271, 2004.

KATZ JJ, CRESPI HL, CZAJKA DM, FINKEL AJ: Course of deuteriation and some physiological effects of deuterium in mice. Am J Physiol 203: 907-913, 1962.

LAISSUE JA, BALLY E, JOEL DD, SLATKIN DN, STONER RD: Protection of mice from whole-body gamma radiation by deuteration of drinking water. Radiat Res 96: 59-64, 1983.

LISITSYN AB, BARYSHEV MG, BASOV AA, BARYSHEVA EV, BYKOV IM, DYDYKIN AS, TEKUTSKAIA EE, TIMAKOV AA, FEDULOVA LV, CHERNUKHA IM, DZHIMAK SS: Influence of deuterium depleted water on the organism of laboratory animals in various functional conditions of nonspecific protective systems (in Russian). Biofizika 59: 757-765, 2014.

MLADIN C, CIOBICA A, LEFTER R, POPESCU A, BILD W: Deuterium-depleted water has stimulating effects on long-term memory in rats. Neurosci Lett 583: 154-158, 2014.

MORI Y, CHIANG S, BENDECK MP, GIACCA A: Insulin decreases atherosclerotic plaque burden and increases plaque stability via nitric oxide synthase in apolipoprotein E-null mice. Am J Physiol Endocrinol Metab 311: E335-E345, 2016. 
PECHANOVA O, SIMKO F: The role of nitric oxide in the maintenance of vasoactive balance. Physiol Res 56 (Suppl 2): S7-S16, 2007.

PECHANOVA O, SIMKO F: Chronic antioxidant therapy fails to ameliorate hypertension: potential mechanisms behind. J Hypertens 27 (Suppl 6): S32-S36, 2009.

PECHANOVA O, ZICHA J, PAULIS L, ZENEBE W, DOBESOVA Z, KOJSOVA S, JENDEKOVA L, SLADKOVA M, DOVINOVA I, SIMKO F, KUNES J: The effect of N-acetylcysteine and melatonin in adult spontaneously hypertensive rats with established hypertension. Eur J Pharmacol 561: 129-136, 2007.

REAVEN GM, CHANG H: Relationship between blood pressure, plasma insulin and triglyceride concentration, and insulin action in spontaneous hypertensive and Wistar-Kyoto rats. Am J Hypertens 4: 34-38, 1991.

SIMKO F, PECHANOVA O, PELOUCH V, KRAJCIROVICOVA K, MULLEROVA M, BEDNAROVA K, ADAMCOVA M, PAULIS L: Effect of melatonin, captopril, spironolactone and simvastatin on blood pressure and left ventricular remodelling in spontaneously hypertensive rats. J Hypertens 27 (Suppl 6): S5-S10, 2009.

SOMLYAI G, MOLNÁR M, LASKAY G, SZABÓ M, BERKÉNYI T, GULLER I, KOVÁCS A: Biological significance of naturally occurring deuterium: the antitumor effect of deuterium depletion. Orv Hetil 151: 1455-1460, 2010.

STEFANESCU I, TITESCU G, TAMAIAN R: Deuterium-depleted water. Effects on living organisms. In: Abstracts of the Third Conference on Isotopic and Molecular Processes. BOGDAN M (ed), 2003.

VRANKOVA S, BARTA A, KLIMENTOVA J, DOVINOVA I, LISKOVA S, DOBESOVA Z, PECHANOVA O, KUNES J, ZICHA J: The regulatory role of nuclear factor kappa B in the heart of hereditary hypertriglyceridemic rat. Oxid Med Cell Longev 2016: 9814038, 2016. doi: 10.1155/2016/9814038.

YU Q, GAO F, MA XL: Insulin says NO to cardiovascular disease. Cardiovasc Res 89: 516-524, 2011.

ZICHA J, PECHÁŇOVÁ O, ČAČÁNYIOVÁ S, CEBOVÁ M, KRISTEK F, TÖRÖK J, ŠIMKO F, DOBEŠOVÁ Z, KUNEŠ J: Hereditary hypertriglyceridemic rat: a suitable model of cardiovascular disease and metabolic syndrome? Physiol Res 55 (Suppl 1): S49-S63, 2006. 